\title{
Quantitative Prediction of Optical Absorption in Molecular Solids from an Optimally Tuned Screened Range-Separated Hybrid Functional
}

\author{
Arun K. Manna, ${ }^{\dagger}$ Sivan Refaely-Abramson, ${ }^{\dagger}$ Anthony M. Reilly, ${ }^{\S}$ Alexandre Tkatchenko, ${ }^{\| \odot}$ \\ Jeffrey B. Neaton, ${ }^{\ddagger, \perp}$ and Leeor Kronik*, ${ }^{\dagger}$ \\ ${ }^{\dagger}$ Department of Materials and Interfaces, Weizmann Institute of Science, Rehovoth 76100, Israel \\ ${ }^{\ddagger}$ Department of Physics, University of California Berkeley, Berkeley, California 94720, United States \\ ${ }^{\S}$ School of Chemical Sciences, Dublin City University, Glasnevin, Dublin 9, Ireland \\ "Physics and Materials Science Research Unit, University of Luxembourg, L-1511 Luxembourg, Luxembourg \\ ${ }^{\perp}$ Molecular Foundry, Lawrence Berkeley National Laboratory, Berkeley, California 94720, United States
}

\section{Supporting Information}

ABSTRACT: We show that fundamental gaps and optical spectra of molecular solids can be predicted quantitatively and nonempirically within the framework of time-dependent density functional theory (TDDFT) using the recently developed optimally tuned screened range-separated hybrid (OT-SRSH) functional approach. In this scheme, the electronic structure of the gas-phase molecule is determined by optimal tuning of the range-separation parameter in a range-separated hybrid functional. Screening and polarization in the solid state are taken into account by adding long-range dielectric screening to the functional form, with the modified functional used to perform self-consistent periodicboundary calculations for the crystalline solid. We provide a comprehensive benchmark for the accuracy of our approach by considering the X23 set of molecular solids and comparing results obtained from TDDFT with those

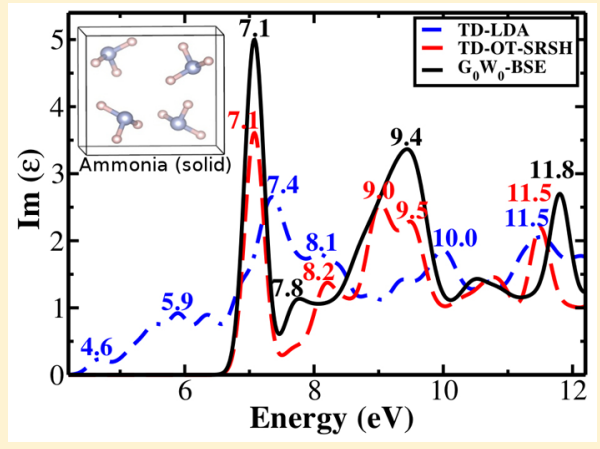
obtained from many-body perturbation theory in the GW-BSE approximation.

We additionally compare results obtained from dielectric screening computed within the random-phase approximation to those obtained from the computationally more efficient many-body dispersion approach and find that this influences the fundamental gap but has little effect on the optical spectra. Our approach is therefore robust and can be used for studies of molecular solids that are typically beyond the reach of computationally more intensive methods.

\section{INTRODUCTION}

The electronic and optical properties of molecular solids have recently attracted significant attention, primarily in the context of optoelectronic devices based on small molecules (see, e.g., refs 1-4). In particular, there is ongoing interest in identifying small-gap organic molecules for high-performance, low-cost, or enhanced-stability optoelectronic devices based on solids comprised of these molecules (see, e.g., refs 5-9 for some recent overviews). Theory can and should play an important role in such investigations, as it can clarify the properties of existing molecular solids and point out promising new ones. $^{10-13}$

Electronic properties, such as the band structure and in particular the transport gap, and optical properties, such as optical absorption in general and the optical gap in particular, are excited-state properties. For inorganic solids, these properties have long been calculated using many-body perturbation theory (MBPT). ${ }^{14-16}$ In MBPT, Dyson's equation is often solved using Hedin's GW approximation, ${ }^{17}$ where $\mathrm{G}$ is the one-particle Green function and $\mathrm{W}$ is the dynamically screened Coulomb interaction. ${ }^{14,18}$ The Bethe-Salpeter equation (BSE) for the two-particle Green function is then solved approximately to predict optical properties. ${ }^{14,19,20}$ In recent years, the GW-BSE approach has been increasingly applied to molecular solids, yielding many important insights (see ref 13 for a recent overview). Unfortunately, such GWBSE calculations can be quite complicated and computationally intensive, limiting our ability to use them routinely, especially in the context of high-throughput calculations for new materials.

Density functional theory (DFT), in both its timeindependent ${ }^{21,22}$ and time-dependent ${ }^{23-26}$ forms, suitable for ground and excited state properties, respectively, is much more computationally efficient. However, common approximations to time-dependent DFT (TDDFT) are known to fail in the solid-state limit. ${ }^{14,27}$ For molecular solids in particular, key quantities, such as the transport gap, the optical gap, and the

Received: October 21, 2017

Published: May 4, 2018 
exciton binding energy (i.e., the difference between the two gaps), are often in qualitative or gross quantitative error. ${ }^{13}$

Recently, Refaely-Abramson et al. have suggested the optimally tuned screened range-separated hybrid (OT-SRSH) functional as a means for quantitative DFT-based prediction of excited-state properties in molecular solids. ${ }^{28,29}$ In this approach, one first computes the underlying gas-phase molecule using an asymptotically correct range-separated hybrid (RSH) functional, in which an optimal range-separation parameter is determined nonempirically, ${ }^{30-33}$ based on satisfaction of the ionization potential theorem. One then uses the same range-separation parameter in the solid-state environment, while accounting explicitly for solid-state polarization by screening the asymptotic potential with a nonempirical dielectric constant. ${ }^{28,29}$

While preliminary results obtained with the OT-SRSH method have shown excellent agreement with GW-BSE data, two important questions remain. First, results have been reported to-date only for a few molecular solids, pentacene, ${ }^{28,29}$ benzene, $\mathrm{C}_{60}{ }^{28}$ and quinacridone ${ }^{34}$ (an air-stable pentacene derivative), with the optical absorption spectrum computed only for pentacene. The validity of the OT-SRSH approach across a wider range of molecular crystals, especially as far as optical properties are concerned, is therefore in need of demonstration. Second, previous OT-SRSH calculations have used the dielectric constant obtained within the random-phase approximation (RPA) for facilitating comparison to MBPT data. However, this step can itself be expensive, and it remains to be seen whether sufficiently accurate results can be obtained from simpler methods for determining the dielectric constant.

In this article, we address both questions by assessing the accuracy of the OT-SRSH approach for transport gaps and optical absorption spectra across the X23 set of molecular solids. $^{35,36}$ This set comprises crystals based on small- to medium-sized organic molecules possessing a variety of weak intermolecular interactions and different degrees of solid-state polarization. It therefore provides a strict benchmark for the OT-SRSH approach. We further compare results obtained using an RPA-based dielectric constant with those obtained using the many-body dispersion (MBD) method. ${ }^{37}$ Within the RPA, we find our OT-SRSH results to be in very good agreement with those obtained from GW for quasiparticle gaps and from GW-BSE for the optical spectrum. We further find that using MBD-based dielectric screening results in larger deviations for quasiparticle gaps but has an essentially negligible effect on the optical absorption, allowing for an inexpensive yet nonempirical prediction of optical properties.

\section{THEORETICAL AND COMPUTATIONAL APPROACH}

Optimally Tuned Range-Separated Hybrid Functionals. In the range-separated hybrid (RSH) method, the Coulomb interaction is range split. Here, we use the rangeseparation scheme suggested by Yanai et al., ${ }^{38}$ which is based on the identity

$$
\frac{1}{r}=\frac{\alpha+\beta \operatorname{erf}(\gamma r)}{r}+\frac{1-[\alpha+\beta \operatorname{erf}(\gamma r)]}{r}
$$

where $r$ is the interelectron coordinate and $\alpha, \beta, \gamma$ are parameters. The full $1 / r$ repulsion is used for the Hartree and correlation terms, but the two terms on the right-hand side of eq 1 are treated differently in the computation of the exchange term. The first term is treated using exact (i.e., Fock) exchange, whereas the second term is treated using local or semilocal exchange. This leads to the following expression for the exchange-correlation energy, $E_{\mathrm{xc}}$ :

$$
\begin{aligned}
E_{\mathrm{xc}}^{\mathrm{RSH}}= & \alpha E_{\mathrm{xx}}^{\mathrm{SR}}+(1-\alpha) E_{\mathrm{DFAx}}^{\mathrm{SR}}+(\alpha+\beta) E_{\mathrm{xx}}^{\mathrm{LR}} \\
& +(1-\alpha-\beta) E_{\mathrm{DFAx}}^{\mathrm{LR}}+E_{\mathrm{DFAc}}
\end{aligned}
$$

where the superscripts "SR" and "LR" denote short-range and long-range contributions, respectively, and the subscripts " $\mathrm{xx}$ ", "DFAx", and "DFAc" denote Fock-like exact exchange, approximate (semi)local exchange, and approximate (semi)local correlation, respectively. ${ }^{39}$ Equation 2 reveals that the parameter $\alpha$ dictates the amount of Fock-like exchange in the short-range $(r \rightarrow 0)$, and the parameter sum $\alpha+\beta$ determines the amount of Fock-like exchange in the long-range $(r \rightarrow \infty)$. The two limits are smoothly interpolated using the error function, with $\gamma$ being the range-separation parameter; i.e., $1 / \gamma$ corresponds to a typical length denoting the transition from SR to LR.

In order to turn eq 2 into a practical functional, one needs to choose the approximate (semi)local exchange-correlation functional and set the parameters $\alpha, \beta$, and $\gamma$. To proceed without introducing empiricism, typical choices for the (semi)local functional would be the local density approximation, $\mathrm{LDA}^{40}$ or the Perdew-Burke-Ernzerhof (PBE) ${ }^{41}$ form of the generalized-gradient approximation (GGA). In some cases, the fraction of SR Fock exchange, $\alpha$, can be determined from first-principles based on the satisfaction of piecewise linearity in fractional DFT, ${ }^{39,42}$ but this is not always possible. ${ }^{43}$ For a wide variety of organic molecules, a universal value of 0.2 has been found to be useful (see, e.g., refs 28, 39, 42-44). This value is used in this work throughout. For any choice of $\alpha$, the condition $\alpha+\beta=1$ guarantess $100 \%$ of LR Fock exchange and therefore the correct asymptotic potential in the gas phase. $^{30,39,42}$

In many popular RSH functionals, the range-separation parameter, $\gamma$, is given a universal value based on fitting against an appropriate data set. ${ }^{38,44-46}$ In the optimal tuning (OT) scheme, $\gamma$ is system dependent but chosen nonempirically. For gas-phase systems, it is obtained by satisfying the ionization potential (IP) theorem, ${ }^{4-50}$ which states that for the exact functional the energy of the highest occupied molecular orbital (HOMO) is equal and opposite to the ionization potential, i.e., $\mathrm{IP}=-\epsilon_{\mathrm{HOMO}}$. Often, this condition is demanded simultaneously for the system in both its neutral and anionic state (where the ionization potential corresponds to the electron affinity of the neutral). ${ }^{30,31,51}$ For any choice of $\alpha$, the optimal tuning scheme then involves the minimization of a target function, $J(\gamma ; \alpha)$, defined by

$$
J^{2}(\gamma ; \alpha)=\sum_{i=N, N+1}\left[\operatorname{IP}^{\gamma ; \alpha}(i)+\varepsilon_{\mathrm{H}}^{\gamma ; \alpha}(i)\right]^{2}
$$

Minimizing this target function has been shown to be equivalent to enforcing piecewise linearity, ${ }^{33,42,52-54}$ resulting in an accurate prediction of the ionization potential and the electron affinity directly from the energy levels of the highest occupied and lowest unoccupied orbitals, respectively. ${ }^{31,33,55}$ Note that use of eq 3 does not require any external reference value for the IP, e.g., from experiment or from wave functionbased calculation. Instead, eq 3 is an internal self-consistency condition between the IP and the HOMO energy.

Refaely-Abramson et al. have suggested that the OT-RSH scheme can be extended to molecular solids by using a screened 
range-separated hybrid (SRSH) ${ }^{28}$ Briefly, in this approach one first selects $\alpha$ and $\gamma$ for the gas-phase molecule as described above. One then notes that in the gas-phase the asymptotic potential is $-1 / r$, but in the solid state, it is $-1 /(\epsilon r)$, where $\epsilon$ is the scalar dielectric constant, i.e., the averaged trace of the dielectric tensor. Therefore, $\beta$ is readjusted to reflect this screening, by demanding that $\alpha+\beta=1 / \epsilon$ instead of $\alpha+\beta=1$ as in the gas phase. The resulting screened RSH functional is then applied to the molecular solid. ${ }^{13,28,29,34}$ Note that this procedure assumes that the effect of anisotropy in the dielectric tensor is negligible and that intramolecular dielectric screening can be neglected during the gas-phase tuning. ${ }^{56}$

X23 Set of Molecular Solids. For evaluating the accuracy of the above approach in a systematic manner, we consider quasiparticle (QP) gaps and optical absorption spectra for the set of 23 noncovalently bound molecular solids, known as the X23 set. ${ }^{35}$ A schematic diagram displaying all molecular entities considered in this set is given in Figure 1. The molecules used

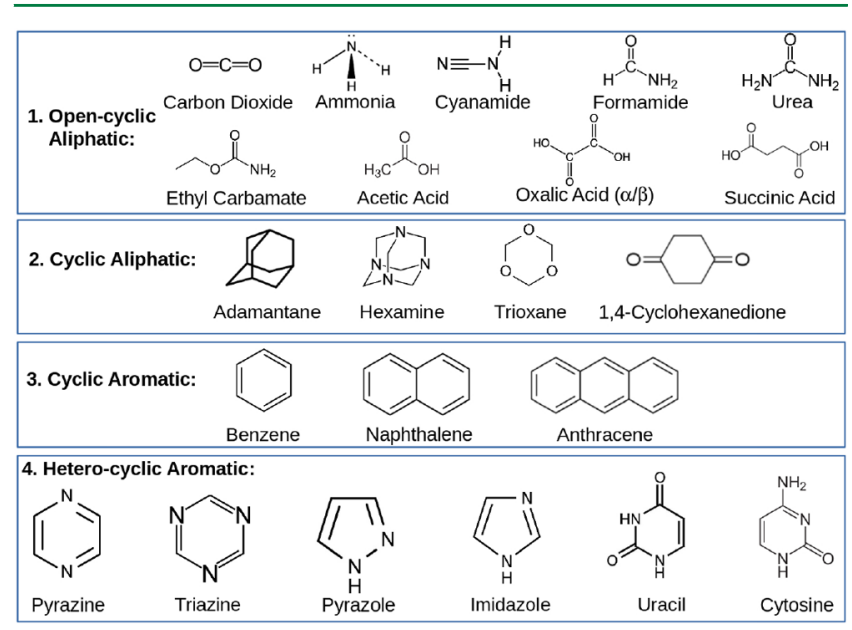

Figure 1. Chemical structures of all organic molecules present in the X23 molecular crystal set.

are small- to medium-sized organic molecules that can be grouped into four subsets based on their chemical identity: open-cyclic aliphatic molecules (carbon dioxide, ammonia, acetic acid, succinic acid, cyanamide, ethyl carbamate, oxalic acid in both $\alpha$ and $\beta$ polymorphs, urea, and formamide), cyclic aliphatic molecules (adamantane, hexamine, trioxane, and 1,4cyclohexane-dione), cyclic aromatic molecules (benzene, naphthalene, and anthracene), and heterocyclic aromatic molecules (cytosine, uracil, triazine, imidazole, pyrazine, and pyrazole). In crystalline solid form, these molecules are weakly bound, typically through $\mathrm{H}$ bonding, $\pi-\pi$ stacking, van der Waals interactions, etc.

Computational Details. All gas-phase OT-RSH calculations presented in this work were based on the LRC- $\omega$ PBE0 RSH functional, ${ }^{44}$ which is based on eq 2 with $\alpha=0.2$ and PBE correlation and short-range exchange components, as implemented in the Q-CHEM code (version 4.3), ${ }^{57}$ but with the range-separated parameter $\gamma$ optimally tuned per system, rather than fixed to its default value. Optimization proceeded via minimization of the target function $J$ given in eq 3 ; i.e., both neutral and anion forms were considered, except for molecules exhibiting an unbound LUMO, where only the neutral form was considered. Optimal values of the range-separated parameter, for all gas-phase molecules used in the X23 set, are given in Table S1 of the Supporting Information. The allelectron cc-pVTZ basis set ${ }^{58}$ was used throughout for all atoms.

All solid-state OT-SRSH calculations were carried out using a modified version of PARATEC (revision 499), ${ }^{59}$ a pseudopotential-planewave code. Here, short-range LDA exchange was used, together with LDA correlation, again with $\alpha=0.2$ throughout. Differences in tuning based on LDA or PBE were found to be insignificant (see refs 28, 29 for more implementation details). LDA-based Troullier-Martins ${ }^{60}$ norm-conserving pseudopotentials, adapted from the ABINIT website, ${ }^{61}$ were used for all atoms. An energy cutoff of $816 \mathrm{eV}$ was used throughout. The number of bands used, as well as details of coarse and fine k-grid meshes used to construct the OT-SRSH wave functions, are given in Table S2 of the Supporting Information. Two different methods were used to evaluate the scalar dielectric constant needed for the determination of $\beta$ in the solid-state calculations. In one, we used the random phase approximation (RPA), as used in the $G_{0} W_{0}$ calculations elaborated below. In the other, we used the framework of the many-body dispersion method, as follows. The dielectric constant has been calculated using the ClausiusMossotti (CM) equation. The required polarizabilities used for evaluating the CM equation were obtained as a unit-cell sum of atomic polarizabilities that incorporate local hybridization effects, as well as electrodynamic screening, as described in ref 62. All calculations of $\epsilon_{\mathrm{MBD}}$ employed the same computational protocol and optimized geometries as in the original X23 benchmark. $^{35}$

For comparison purposes, all molecular solids were also computed using a standard one-shot perturbative $G_{0} W_{0}$ calculation, ${ }^{13,18}$ based on the DFT eigenvalues and eigenvectors obtained from an LDA calculation within PARATEC. We used a generalized plasmon pole model, ${ }^{18}$ implemented within the BerkeleyGW package (trunk version, revision 6539). ${ }^{63}$ This approach has previously been established as a quantitatively useful tool for the study of molecular solids (see ref 13, and references therein). The dielectric function and the self-energy were computed using a large number of unoccupied states, as listed in Table S3 of the Supporting Information.

Optical spectra in the solid state were computed using TDDFT with the LDA and the OT-SRSH functional, as well as with the Bethe-Salpeter equation (BSE) based on the $G_{0} W_{0}$ output. Both TDDFT and BSE calculations were performed using the BerkeleyGW package, ${ }^{63}$ modified to include TDDFT, with incident light polarization averaged over the main unit-cell axes. The kernel was calculated on a coarse wave function grid, then interpolated to a fine grid using the interpolation scheme suggested by Rohlfing and Louie, ${ }^{64}$ except for a few crystals with a very large unit cells, for which only a coarse grid was used. We used a slightly shifted grid to generate the transition matrix elements in the dielectric function, using a velocity operator to approximate an incident light along a specific direction. ${ }^{64}$ Grid shift directions were set along the $a, b$, and $c$ unit-cell axes. The number of occupied and unoccupied states used to construct the kernel matrix is provided in Table S4 of the Supporting Information.

\section{RESULTS AND DISCUSSION}

Fundamental Gap. We begin our benchmark evaluation by comparing the fundamental gaps computed using OT-SRSH and $G_{0} W_{0}$ methods for all solids in the X23 set. For consistency, all OT-SRSH results presented are based on the dielectric constant obtained within RPA, except in the last part 
of this section, where comparison with MBD-based results is explicitly made. With both OT-SRSH and GW, we computed the fundamental gap as the energy difference between the highest occupied and lowest unoccupied state at the $\Gamma$ point of the Brillouin zone, as the intermolecular orbital hybridization and therefore band dispersion are very small.

OT-SRSH- and GW-computed fundamental gaps are given in Table 1, where they are additionally compared to LDA-

Table 1. Fundamental Gaps of X23 Set of Molecular Solids (in eV), Calculated Using LDA, OT-SRSH, and $G_{0} W_{0}$, Additionally Compared to Gas-Phase Fundamental Gaps Calculated Using OT-RSH

\begin{tabular}{|c|c|c|c|c|}
\hline \multirow[b]{2}{*}{ Molecular Solid } & \multicolumn{3}{|c|}{ Fundamental Gap $\left(E_{\mathrm{g}}\right)$} & \multirow[b]{2}{*}{$G_{0} W_{0}$} \\
\hline & LDA & OT-RSH & OT-SRSH & \\
\hline Carbon dioxide & 6.4 & 13.9 & 11.2 & 11.2 \\
\hline Ammonia & 4.3 & 10.7 & 7.9 & 7.7 \\
\hline Cyanamide & 4.6 & 10.8 & 8.0 & 8.0 \\
\hline Formamide & 4.9 & 10.7 & 8.8 & 8.8 \\
\hline Urea & 4.8 & 10.0 & 8.0 & 7.9 \\
\hline Ethyl carbamate & 5.6 & 10.3 & 9.0 & 8.8 \\
\hline Acetic acid & 5.1 & 10.8 & 9.1 & 9.3 \\
\hline Oxalic acid $(\alpha)$ & 3.2 & 11.5 & 6.7 & 6.9 \\
\hline Oxalic acid $(\beta)$ & 3.5 & 11.5 & 7.3 & 7.5 \\
\hline Succinic acid & 5.2 & 10.7 & 9.1 & 9.1 \\
\hline Adamantane & 4.8 & 9.7 & 7.5 & 7.6 \\
\hline Hexamine & 5.0 & 8.3 & 7.5 & 7.8 \\
\hline Trioxane & 5.9 & 10.7 & 9.7 & 9.5 \\
\hline 1,4-Cyclohexane-dione & 3.5 & 10.0 & 7.0 & 7.0 \\
\hline Benzene & 4.3 & 9.3 & 6.8 & 6.9 \\
\hline Naphthalene & 3.2 & 8.1 & 5.2 & 5.4 \\
\hline Anthracene & 2.1 & 6.8 & 3.9 & 4.1 \\
\hline Pyrazine & 2.8 & 10.0 & 6.2 & 6.0 \\
\hline Triazine & 3.0 & 10.6 & 6.2 & 6.3 \\
\hline Pyrazole & 4.8 & 9.4 & 7.6 & 8.1 \\
\hline Imidazole & 4.8 & 8.9 & 7.6 & 7.9 \\
\hline Uracil & 3.4 & 9.5 & 6.4 & 6.4 \\
\hline Cytosine & 3.4 & 8.8 & 6.1 & 6.1 \\
\hline
\end{tabular}

computed gaps and to gas-phase OT-RSH gaps. As expected, the gas-phase gaps are substantially larger than the solid-state ones (by as little as $0.8 \mathrm{eV}$ and as much as $4.8 \mathrm{eV}$ ). This reflects the well-known phenomena of polarization-induced gaprenormalization in molecular solids, ${ }^{65}$ which is clearly captured in the OT-SRSH scheme ${ }^{28}$ but is known to be absent in standard functionals. ${ }^{13,66}$ It is readily observed that the OTSRSH gaps agree very well indeed with the GW ones. The deviation between the gaps computed with GW and OT-SRSH is summarized graphically in Figure 2. The differences are usually $0.2 \mathrm{eV}$ at most, with a mean absolute deviation of only $0.15 \mathrm{eV}$. Only two solids (hexamine and imidazole) exhibited a somewhat larger deviation of $0.3 \mathrm{eV}$ and only one solid (pyrazole) exhibits a larger deviation of $0.5 \mathrm{eV}$. Not surprisingly, these gaps are substantially larger than those obtained with LDA, which is well known to underestimate fundamental gaps in general. ${ }^{14,18}$

The above results establish dielectric screening as key to accurate treatment of molecular solids. This observation suggests several additional comments. First, there is growing

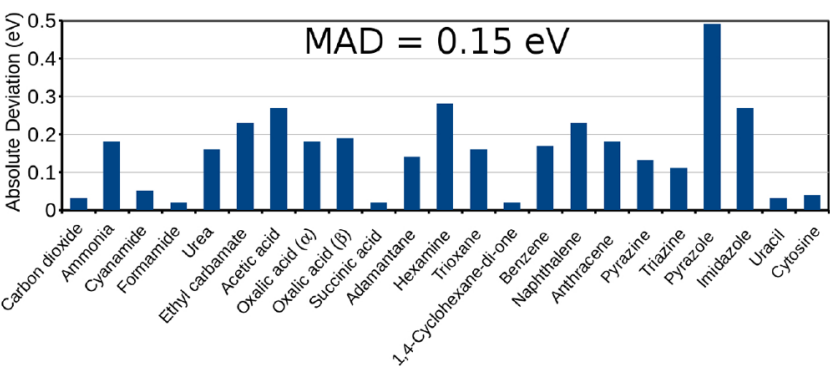

Figure 2. Absolute deviations and mean absolute deviation (MAD) in the calculated quasiparticle gap between the OT-SRSH and $G_{0} W_{0}$ for the X23 set of molecular solids. For OT-SRSH calculations, the $G_{0} W_{0^{-}}$ computed RPA macroscopic dielectric constant $\left(\epsilon^{\mathrm{RPA}}\right)$ is used.

recent interest in solid-state screening as an ingredient in the construction of density functionals in general (see, e.g., 29, 67-71). Specifically for molecular solids, there is growing interest in embedding a range-separated hybrid molecular calculation within a polarizable continuum model (PCM) to mimic solid-state effects (see, e.g., 56, 72-74, and references therein). We note that the optimal tuning of $\gamma$ can be performed within the PCM also in the absence of a screening term in the functional, i.e., with the full asymptotic potential. While this too leads to improved predictions for fundamental gaps, it usually comes at the cost of a greatly reduced rangeseparation parameter, which may affect other system properties, and does not contain a full physical description of dielectric screening. For a complete discussion, see refs 56, 74, and 75 . Additionally, it is important to note that inclusion of a dielectric constant renormalizes the fundamental gap even in a gas-phase calculation. $^{56,74}$ This is still a manifestation of bulk polarization, as the value of the dielectric constant must be supplied by a bulk calculation or a PCM-based calculation. Beyond capturing polarization, our approach, which is based on a full solid-state calculation, also captures intermolecular dispersion effects. ${ }^{28}$

Optical Absorption Spectra. We next compare the optical absorption spectra calculated using TD-OT-SRSH with those obtained from the $G_{0} W_{0}$-BSE methods. As we are interested in allowed optical transitions, we only consider singlet excitations. The complete set of optical absorption spectra is shown in Figures 3 and 4, for solids based on aliphatic and aromatic molecules, respectively. In addition, the energy of the lowest singlet excitation and the position of lowest-lying main optical absorption peak are provided in Table 2, along with experimental values for comparison, where available. Additional comparison between the lowest solid-state OT-SRSH and gasphase OT-RSH energies can be found in Table S5 of the SI. Generally, the optical gap is not as strongly affected by the transition from the gas phase to the solid state as the fundamental gap, which is expected given that the optical gap corresponds to a neutral excitation that is less sensitive to dielectric screening (an issue we return to below). However, the complete optical spectrum may be very different, as demonstrated for ammonia in Figure S1 of the SI. Therefore, the following discussion focuses on the solid-state results.

It is readily observed from Figures 3 and 4 that absorption spectra computed with the TD-OT-SRSH approach do indeed agree well with those computed using $G_{0} W_{0}$-BSE, across the board, over a range of several eV. Specifically, the position of intense peaks, found by the two approaches, typically agrees within $\sim 0.2-0.3 \mathrm{eV}$, which is excellent given an accuracy of $\sim 0.1 \mathrm{eV}$ at best for either approach separately. This observation 

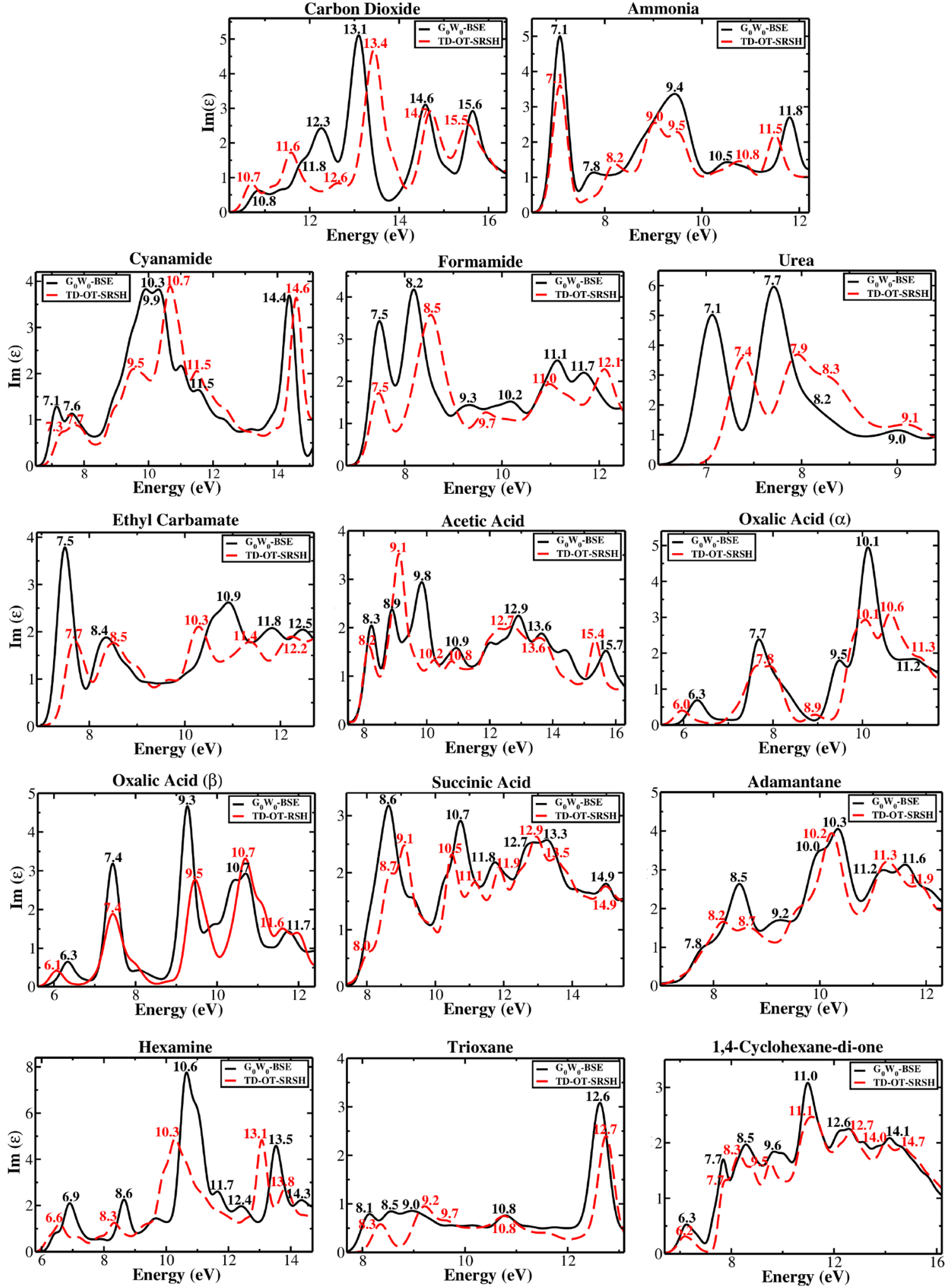

Figure 3. Imaginary part of the dielectric function of aliphatic-molecule-based solids in the X23 data set, calculated using $G_{0} W_{0} / \mathrm{BSE}$ (black solid lines) and TD-OT-SRSH (red dashed lines).

is quantified in Figure 5, which shows deviations between peak positions using TD-OT-SRSH and GW-BSE, for the lowestenergy peak (left) and for all peaks shown in Figures 3 and 4 (right). The mean absolute deviation is only $0.2 \mathrm{eV}$ for either the lowest-energy peak or all shown peaks, with deviations rarely exceeding $0.3 \mathrm{eV}$.
As expected based on known shortcomings of TDLDA, ${ }^{13,14,27}$ the TD-OT-SRSH data offer an improvement over TDLDA data that is not only quantitative but also qualitative. This is demonstrated in Figure 6 for two representative cases: the ammonia and uracil solids. For the ammonia, TDLDA produces an extended spurious absorption 

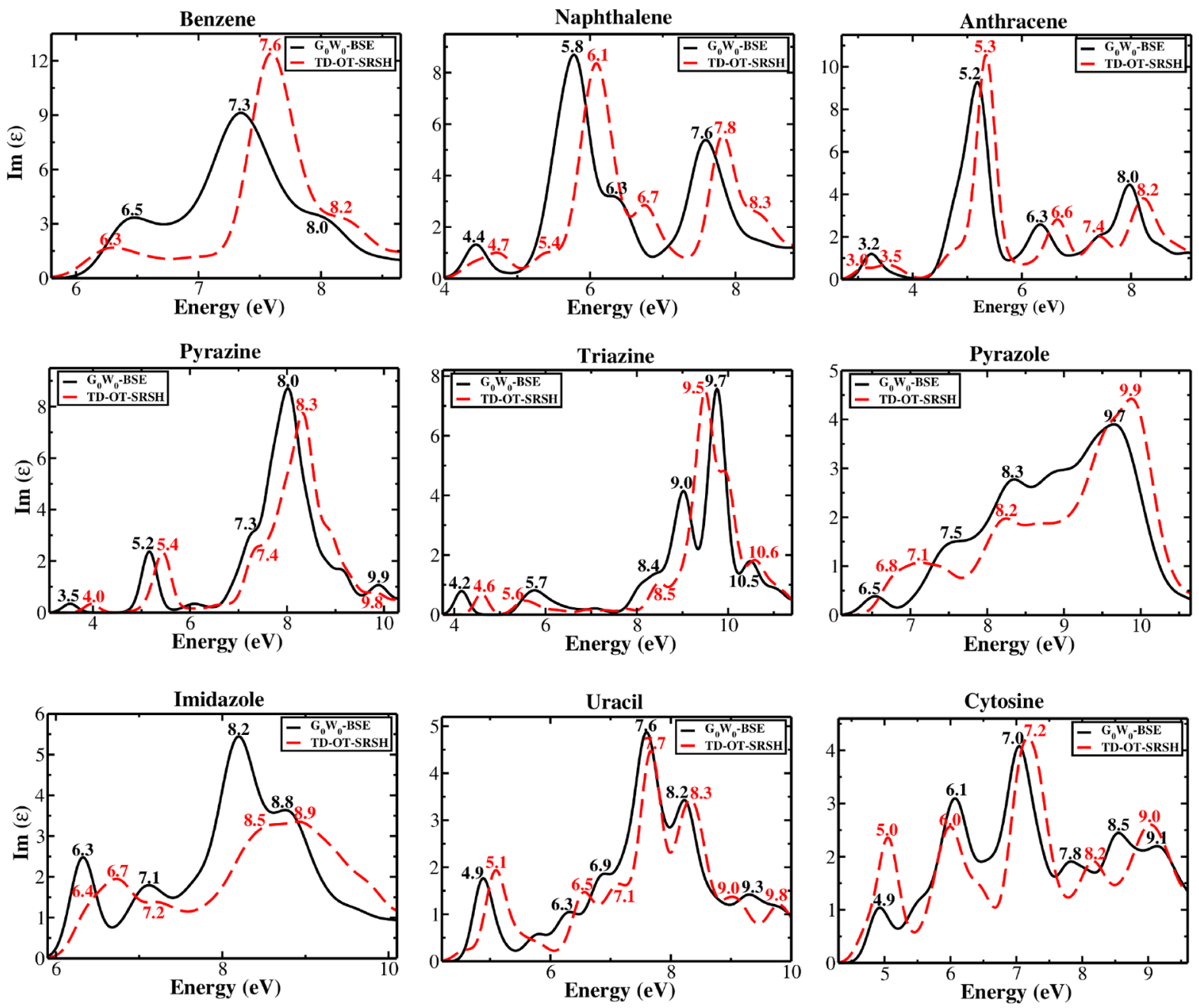

Figure 4. Imaginary part of the dielectric function of aromatic-molecule-based solids in the X23 data set, calculated using $G_{0} W_{0} / \mathrm{BSE}$ (black solid lines) and TD-OT-SRSH (red dashed lines).

"tail", starting at $\sim 4.6 \mathrm{eV}$, whereas both TD-OT-SRSH and GW-BSE predict a sharp onset of absorption at $\sim 7.1 \mathrm{eV}$. The same phenomenon has been previously observed for a nonmolecular solid: $\mathrm{LiF}^{29}$ For uracil, TDLDA (as well as $\mathrm{TDPBE}^{87}$ ) produces a spurious peak at $\sim 3.8 \mathrm{eV}$. Further analysis (not shown for brevity) reveals that this peak results from LDA misordering of the HOMO and HOMO-1 orbitals, which is remedied by the OT-SRSH calculation and removes the false peak.

In many of the molecular solids, the lowest singlet excitation possesses a small matrix element and contributes little to the optical spectrum. This is reflected in Table 2, where the energy of the first-excited state is often predicted to be quite different from the energy of the lowest-lying absorption peak, using either TD-OT-SRSH or GW-BSE. We found that larger differences between the two methods often, but not always, arise for these low-absorption excitations, despite the excellent agreement in predictions of the fundamental gap and the highabsorption excitations. As an example, for ammonia, the lowestenergy singlet excitation is predicted to be 6.7 or $6.6 \mathrm{eV}$ using TD-OT-SRSH or GW-BSE, respectively, with both values in excellent agreement with the experimental values of $6.6 \mathrm{eV} .{ }^{77} \mathrm{~A}$ similar picture emerges for adamantane. But for urea or 1,4cyclohexane-dione, the difference between the two predictions is a much larger and entirely non-negligible $0.6 \mathrm{eV}$. We note that these lowest-lying transitions often involve transitions between highly localized orbitals, which can exhibit large selfinteraction errors. Therefore, the discrepancy may be due to remaining issues in the TDDFT calculation but may well be also due to LDA being an insufficient starting point for the GW-BSE calculation. ${ }^{88-90}$ We note in passing that starting point issues and TD-OT-SRSH inaccuracies can be observed also in the context of delocalized orbitals, e.g., for benzene and oligoacene molecules and solids, ${ }^{29,43,90-92}$ but this is an issue separate from the optical absorption differences seen here.

Finally, we note that Table 2 and Table S5 additionally show that in some case absorption peak positions revealed in the solid state can differ meaningfully from those obtained in the gas phase with TD-OT-RSH. This reflects the fact that owing to orbital localization or delocalization, band dispersion, and possible charge transfer, the nature of the solid-state exciton can be very different from that of the gas-phase one. ${ }^{13,93,94}$

Effect of Dielectric Constant. To facilitate comparison to GW-BSE, which relies on evaluation of the dielectric function using the random-phase approximation (RPA), all OT-SRSH and TD-OT-SRSH results reported above were obtained using $\epsilon^{\mathrm{RPA}}$. Here, we explore the effect of basing the calculation on an evaluation of the dielectric constant using the inexpensive many-body dispersion (MBD) method. ${ }^{37} \mathrm{~A}$ comparison of dielectric constants and quasiparticle gaps obtained from using 
Table 2. Energy of Lowest Singlet Excitation and Position of Lowest-Lying Main Optical Absorption Peak, Calculated Using TD-OT-SRSH and $G_{0} W_{0}$-BSE for the X23 Set of Molecular Solids and Additionally Compared to Experimental Values (where available $)^{a}$

\begin{tabular}{|c|c|c|c|c|c|c|c|}
\hline \multirow[b]{2}{*}{ Molecular Solid } & \multicolumn{4}{|c|}{ First Excited State $\left(S_{1}\right)$ Energy } & \multicolumn{3}{|c|}{ Optical Peak Position } \\
\hline & TD-LDA & TD-SRSH & $G_{0} W_{0}$-BSE & Expt. & TD-LDA & TD-SRSH & $G_{0} W_{0}-\mathrm{BSE}$ \\
\hline Carbon dioxide & 6.4 & 8.9 & 8.3 & $8.9^{76}$ & 7.2 & 10.7 & 10.8 \\
\hline Ammonia & 4.3 & 6.7 & 6.6 & $6.6^{77}$ & 4.6 & 7.1 & 7.1 \\
\hline Cyanamide & 4.6 & 6.0 & 5.4 & - & 5.6 & 7.3 & 7.1 \\
\hline Formamide & 4.5 & 5.9 & 5.4 & - & 5.3 & 7.5 & 7.5 \\
\hline Urea & 4.8 & 7.1 & 6.5 & $6.2^{78}$ & 6.4 & 7.4 & 7.1 \\
\hline Ethyl carbamate & 5.6 & 7.3 & 6.5 & - & 7.2 & 7.7 & 7.5 \\
\hline Acetic acid & 5.1 & 5.8 & 5.6 & - & 5.5 & 8.2 & 8.3 \\
\hline Oxalic acid $(\alpha)$ & 3.2 & 4.7 & 4.4 & - & 3.7 & 6.0 & 6.3 \\
\hline Oxalic acid $(\beta)$ & 3.4 & 4.8 & 4.5 & - & 3.7 & 6.1 & 6.3 \\
\hline Succinic acid & 5.0 & 6.2 & 5.7 & - & 5.4 & 8.7 & 8.6 \\
\hline Adamantane & 4.8 & 6.8 & 6.8 & $6.5^{79}$ & 6.8 & 8.2 & 8.5 \\
\hline Hexamine & 4.7 & 6.0 & 6.2 & - & 5.4 & 6.6 & 6.9 \\
\hline Trioxane & 5.8 & 8.2 & 7.9 & - & 6.3 & 8.3 & 8.1 \\
\hline 1,4-Cyclohexane-dione & 3.2 & 4.5 & 3.9 & - & 3.9 & 6.2 & 6.3 \\
\hline Benzene & 4.3 & 5.4 & 4.9 & $4.7^{80}$ & 4.9 & 6.3 & 6.5 \\
\hline Naphthalene & 3.1 & 4.2 & 4.1 & $3.9^{81}$ & 3.3 & 4.7 & 4.4 \\
\hline Anthracene & 2.0 & 2.9 & 3.2 & $3.1^{82}$ & 2.1 & 3.0 & 3.2 \\
\hline Pyrazine & 2.7 & 4.0 & 3.5 & $3.8^{83}$ & 3.0 & 4.0 & 3.5 \\
\hline Triazine & 2.8 & 4.4 & 3.9 & $3.7^{84}$ & 3.4 & 4.6 & 4.2 \\
\hline Pyrazole & 4.8 & 6.7 & 6.5 & - & 5.5 & 6.8 & 6.5 \\
\hline Imidazole & 4.5 & 6.3 & 6.3 & - & 5.0 & 6.4 & 6.3 \\
\hline Uracil & 3.4 & 4.6 & 4.9 & $4.5^{85}$ & 3.8 & 5.1 & 4.9 \\
\hline Cytosine & 3.4 & 4.7 & 4.9 & $4.4^{86}$ & 3.8 & 5.0 & 4.9 \\
\hline
\end{tabular}

${ }^{a}$ All energies are in $\mathrm{eV}$.
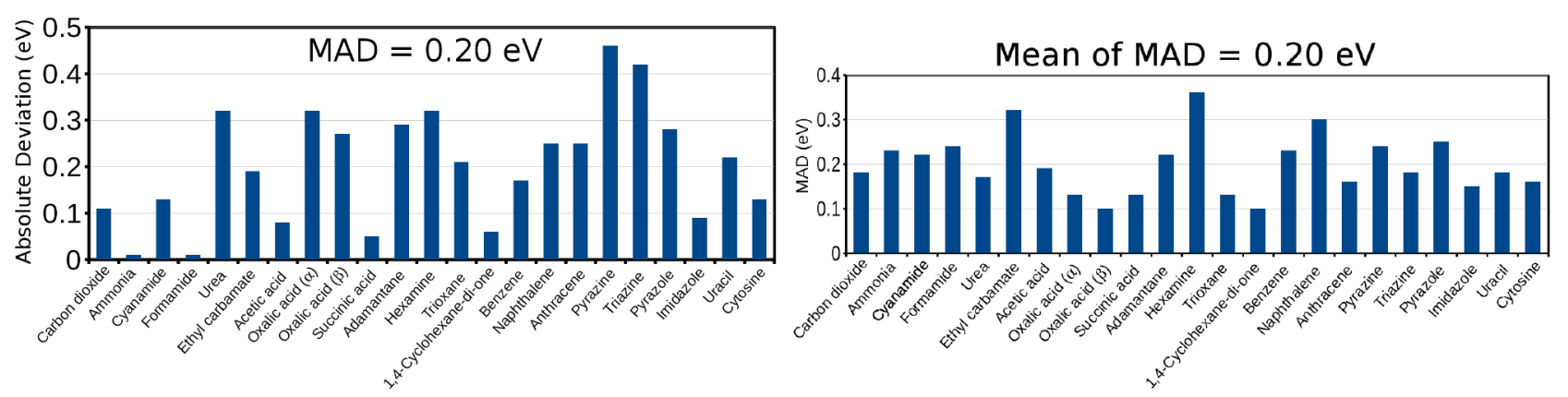

Figure 5. Absolute deviations between the TD-OT-SRSH- and $G_{0} W_{0}$-BSE-computed peak positions in the optical spectra of the X23 molecular solid set. (Left) Lowest peak position. (Right) Mean absolute deviations of all peaks shown in Figure 3 or 4.
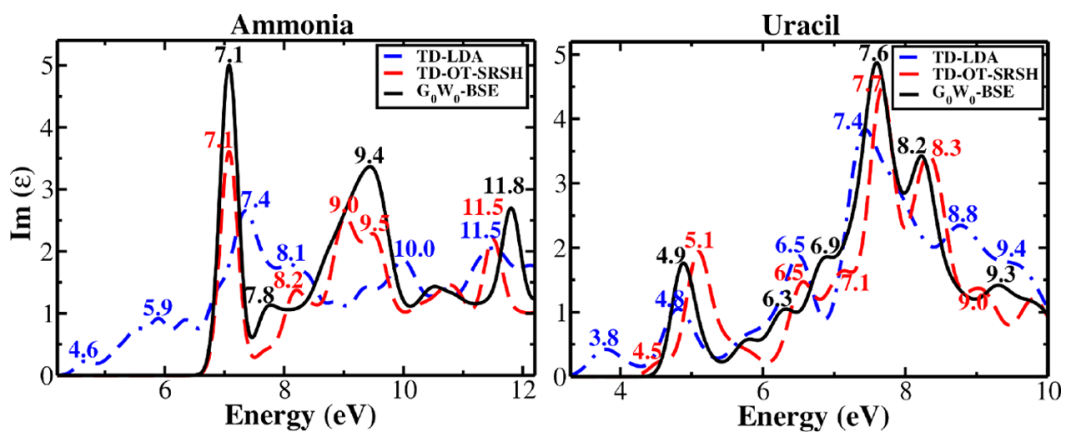

Figure 6. Imaginary part of the dielectric function of the ammonia and uracil molecular solids, calculated using $G_{0} W_{0} / \mathrm{BSE}$ (black solid lines), TDOT-SRSH (red dashed lines), and TDLDA (dotted blue lines). 

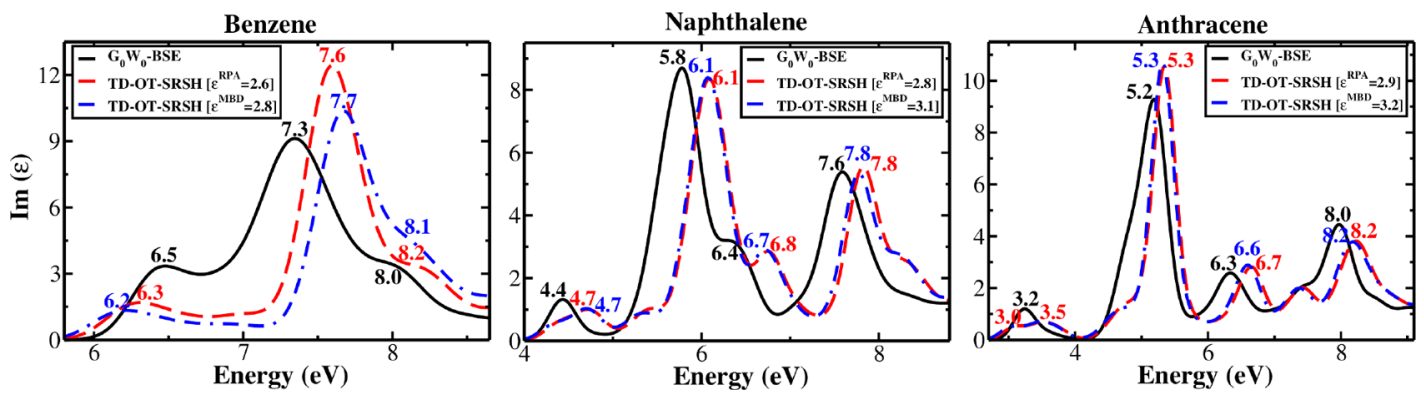

Figure 7. The imaginary part of the dielectric function for the aromatic acene molecular solids (benzene, naphthalene, and anthracene), calculated using $G_{0} W_{0} /$ BSE (black solid lines), TD-OT-SRSH based on $\epsilon^{\mathrm{RPA}}$ (red dashed lines)], and TD-OT-SRSH based on $\epsilon^{\mathrm{MBD}}$ (blue dash-dotted lines). The different $\epsilon$ values are denoted in the figure.

(the averaged trace of) $\epsilon^{\mathrm{RPA}}$ and $\epsilon^{\mathrm{MBD}}$ is given in Table $\mathrm{S} 6$ of the Supporting Information. We find that $\epsilon^{\mathrm{MBD}} \geq \epsilon^{\mathrm{RPA}}$ throughout the X23 set, possibly because it is computed based on PBE, which tends to overestimate polarizabilities. Therefore, the MBD-computed fundamental gaps are generally smaller than RPA-computed ones. While the difference is often small, it can be substantial, as much as $0.8 \mathrm{eV}$ for succinic acid and $0.7 \mathrm{eV}$ for uracil. It would be interesting for future work to examine whether a self-consistent $\epsilon$ value, obtained from MBD calculations based on the OT-SRSH results, would result in an improved agreement.

While the differences in the dielectric constant do affect the fundamental gap, their effect on the optical spectra is much smaller. This is reasonable, as the fundamental gap reflects charged excitations, whereas optical excitations are neutral. The effect of the dielectric constant on the TD-OT-SRSH absorption spectra is demonstrated in Figure 7 for the case of the acene-based molecular solids. Clearly, the effect of $\epsilon$ is marginal (e.g., differences of $\sim 0.1 \mathrm{eV}$ at most in the absorption peak position for the benzene solid). The effect on the lowest singlet-excitation energy is equally small. Therefore, using MBD dielectric constants leads to an inexpensive and predictive calculation of optical spectra in molecular solids.

\section{CONCLUSIONS}

In conclusion, we have computed fundamental gaps and optical spectra for the entire X23 benchmark set of molecular solids using the recently developed optimally tuned screened rangeseparated hybrid functional approach. In this two-stage approach, optimal tuning of a range-separated hybrid functional is first used for an accurate and predictive calculation of the gasphase electronic structure. Dielectric screening is then built into the functional to obtain a self-consistent prediction for the solid-state electronic structure and optical properties. The obtained results have been compared to many-body perturbation theory calculations within the GW-BSE approach. Agreement has been found to be very good to excellent throughout, with somewhat larger differences possible for optically dark singlet excitations that do not affect the optical spectrum. Furthermore, we have shown that inexpensive evaluation of the dielectric constant using many-body dispersion is sufficient for obtaining accurate optical spectra, opening the door to a low-cost, fully predictive calculation of optical spectra in molecular solids.

\section{ASSOCIATED CONTENT}

\section{Supporting Information}

The Supporting Information is available free of charge on the ACS Publications website at DOI: 10.1021/acs.jctc.7b01058.

Gas-phase optimal range-separation parameters $(\gamma)$; coarse and fine k-grid meshes used for the LDA, OTSRSH, and $G_{0} W_{0}$ calculations; number of unoccupied electronic bands used to construct the dielectric function and self-energy in the $G_{0} W_{0}$ calculations; number of occupied and unoccupied states used to construct the BSE and TDDFT kernel matrix; RPA and MBD dielectric constants and their corresponding OT-SRSH gaps. (PDF)

\section{AUTHOR INFORMATION}

\section{Corresponding Author}

*E-mail: leeor.kronik@weizmann.ac.il.

ORCID

Sivan Refaely-Abramson: 0000-0002-7031-8327

Alexandre Tkatchenko: 0000-0002-1012-4854

Leeor Kronik: 0000-0001-6791-8658

Notes

The authors declare no competing financial interest.

\section{ACKNOWLEDGMENTS}

A.K.M. gratefully acknowledges the VATAT postdoctoral fellowship awarded by the Government of Israel and the Weizmann Institute of Science for research support. L.K. acknowledges support of the US Air Force and the NSF-BSF program. Portions of this work were supported by the National Science Foundation under Grant No. DMR-1708892. Work at the Molecular Foundry was supported by the Office of Science, Office of Basic Energy Sciences, U.S. Department of Energy under Contract No. DE-AC02-05CH11231. The authors thank NERSC for providing computational facilities.

\section{REFERENCES}

(1) Forrest, S. R.; Thompson, M. E. Introduction: Organic Electronics and Optoelectronics. Chem. Rev. 2007, 107, 923-925.

(2) Kaur, N.; Singh, M.; Pathak, D.; Wagner, T.; Nunzi, J. Organic materials for photovoltaic applications: Review and mechanism. Synth. Met. 2014, 190, 20-26.

(3) Baeg, K.-J.; Binda, M.; Natali, D.; Caironi, M.; Noh, Y.-Y. Organic Light Detectors: Photodiodes and Phototransistors. Adv. Mater. 2013, $25,4267-4295$

(4) Hu, W.; Bai, F.; Gong, X.; Zhan, X.; Fu, H.; Bjornholm, T. Organic Optoelectronics, 1st ed.; Wiley-VCH, Heidelberg, 2013. 
(5) Li, X.-C.; Wang, C.-Y.; Lai, W.-Y.; Huang, W. Triazatruxenebased materials for organic electronics and optoelectronics. J. Mater. Chem. C 2016, 4, 10574-10587.

(6) Dong, J.-X.; Zhang, H.-L. Azulene-based organic functional molecules for optoelectronics. Chin. Chem. Lett. 2016, 27, 1097-1104.

(7) Parker, T. C.; Patel, D. G. D.; Moudgil, K.; Barlow, S.; Risko, C.; Brédas, J.-L.; Reynolds, J. R.; Marder, S. R. Heteroannulated acceptors based on benzothiadiazole. Mater. Horiz. 2015, 2, 22-36.

(8) Li, D.; Zhang, H.; Wang, Y. Four-coordinate organoboron compounds for organic light-emitting diodes (OLEDs). Chem. Soc. Rev. 2013, 42, 8416-8433.

(9) Lucas, B.; Trigaud, T.; Videlot-Ackermann, C. Organic transistors and phototransistors based on small molecules. Polym. Int. 2012, 61, 374-389.

(10) Brédas, J.-L.; Norton, J. E.; Cornil, J.; Coropceanu, V. Molecular Understanding of Organic Solar Cells: The Challenges. Acc. Chem. Res. 2009, 42, 1691-1699.

(11) Savoie, B. M.; Jackson, N. E.; Marks, T. J.; Ratner, M. A Reassessing the use of one-electron energetics in the design and characterization of organic photovoltaics. Phys. Chem. Chem. Phys. 2013, 15, 4538-4547.

(12) Risko, C.; Brédas, J.-L. In Multiscale Modelling of Organic and Hybrid Photovoltaics; Beljonne, D., Cornil, J., Eds.; Springer: Berlin, 2014; pp 1-38.

(13) Kronik, L.; Neaton, J. B. Excited-State Properties of Molecular Solids from First Principles. Annu. Rev. Phys. Chem. 2016, 67, 587616.

(14) Onida, G.; Reining, L.; Rubio, A. Electronic Excitations: Density-Functional versus many-Body Green's-Function Approaches. Rev. Mod. Phys. 2002, 74, 601.

(15) Strinati, G. Application of the Green's functions method to the study of the optical properties of semiconductors. Riv. Nuovo Cimento 1988, 11, 1-86.

(16) Aulbur, W. G.; Jonsson, L.; Wilkins, J. W. Quasiparticle calculations in solids. Solid State Phys. 2000, 54, 1-218.

(17) Hedin, L. New Method for Calculating the One-Particle Green's Function with Application to the Electron-Gas Problem. Phys. Rev. 1965, 139, A796.

(18) Hybertsen, M.; Louie, M. Electron Correlation in Semiconductors and Insulators: Band Gaps and Quasiparticle Energies. Phys. Rev. B: Condens. Matter Mater. Phys. 1986, 34, 5390.

(19) Rohlfing, M.; Louie, S. G. Electron-Hole Excitations in Semiconductors and Insulators. Phys. Rev. Lett. 1998, 81, 2312-2315.

(20) Albrecht, S.; Reining, L.; Del Sole, R.; Onida, G. Ab Initio Calculation of Excitonic Effects in the Optical Spectra of Semiconductors. Phys. Rev. Lett. 1998, 80, 4510-4513.

(21) Gross, E. K. U.; Dreizler, R. M. Density Functional Theory; Springer, 1995.

(22) Parr, R. G.; Yang, W. Density Functional Theory of Atoms and Molecules; Oxford University Press: Oxford, 1989.

(23) Marques, M. A.; Maitra, N. T.; Nogueira, F. M.; Gross, E. K. U.; Rubio, A. Fundamentals of Time-Dependent Functional Theory; Springer: Berlin, 2012; Vol. 837; pp 1-559.

(24) Casida, M. E. In in Recent Advances in Density-Functional Methods Part I; Chong, D. P., Ed.; World Scientific: Singapore, 1995; pp 1155.

(25) Burke, K.; Werschnik, J.; Gross, E. K. U. Time-dependent density functional theory: Past, present, and future. J. Chem. Phys. 2005, 123, 062206-062214.

(26) Ullich, C. A. Time-Dependent Density-Functional Theory: Concepts and Applications; Oxford University Press: Oxford, 2012.

(27) Maitra, N. T. Perspective: Fundamental Aspects of TimeDependent Density Functional Theory. J. Chem. Phys. 2016, 144, 220901-220919.

(28) Refaely-Abramson, S.; Sharifzadeh, S.; Jain, M.; Baer, R.; Neaton, J. B.; Kronik, L. Gap renormalization of molecular crystals from density-functional theory. Phys. Rev. B: Condens. Matter Mater. Phys. 2013, 88, 081204-081208.
(29) Refaely-Abramson, S.; Jain, M.; Sharifzadeh, S.; Neaton, J. B.; Kronik, L. Solid-state optical absorption from optimally tuned timedependent range-separated hybrid density functional theory. Phys. Rev. B: Condens. Matter Mater. Phys. 2015, 92, 081204-081209.

(30) Stein, T.; Kronik, L.; Baer, R. Reliable Prediction of Charge Transfer Excitations in Molecular Complexes Using Time-Dependent Density Functional Theory. J. Am. Chem. Soc. 2009, 131, 2818-2820.

(31) Stein, T.; Eisenberg, H.; Kronik, L.; Baer, R. Fundamental Gaps in Finite Systems from Eigenvalues of a Generalized Kohn-Sham Method. Phys. Rev. Lett. 2010, 105, 266802-266805.

(32) Refaely-Abramson, S.; Baer, R.; Kronik, L. Fundamental and excitation gaps in molecules of relevance for organic photovoltaics from an optimally tuned range-separated hybrid functional. Phys. Rev. B: Condens. Matter Mater. Phys. 2011, 84, 075144-075151.

(33) Kronik, L.; Stein, T.; Refaely-Abramson, S.; Baer, R. Excitation Gaps of Finite-Sized Systems from Optimally Tuned Range-Separated Hybrid Functionals. J. Chem. Theory Comput. 2012, 8, 1515-1531.

(34) Lüftner, D.; Refaely-Abramson, S.; Pachler, M.; Resel, R.; Ramsey, M. G.; Kronik, L.; Puschnig, P. Phys. Rev. B: Condens. Matter Mater. Phys. 2014, 90, 075204-075213.

(35) Reilly, A. M.; Tkatchenko, A. Understanding the role of vibrations, exact exchange, and many-body van der Waals interactions in the cohesive properties of molecular crystals. J. Chem. Phys. 2013, $139,024705$.

(36) Otero-de-la Roza, A.; Johnson, E. R. A benchmark for noncovalent interactions in solids. J. Chem. Phys. 2012, 137, 054103054112 .

(37) Tkatchenko, A.; DiStasio, R. A.; Car, R.; Scheffler, M. Accurate and Efficient Method for Many-Body van der Waals Interactions. Phys. Rev. Lett. 2012, 108, 236402-236406.

(38) Yanai, T.; Tew, D. P.; Handy, N. C. A new hybrid exchangecorrelation functional using the Coulomb-attenuating method (CAMB3LYP). Chem. Phys. Lett. 2004, 393, 51-57.

(39) Refaely-Abramson, S.; Sharifzadeh, S.; Govind, N.; Autschbach, J.; Neaton, J. B.; Baer, R.; Kronik, L. Quasiparticle Spectra from a Nonempirical Optimally Tuned Range-Separated Hybrid Density Functional. Phys. Rev. Lett. 2012, 109, 226405-226408.

(40) Kohn, W.; Sham, L. J. Phys. Rev. 1965, 140, A1133.

(41) Perdew, J. P.; Burke, K.; Ernzerhof, M. Generalized Gradient Approximation Made Simple. Phys. Rev. Lett. 1996, 77, 3865-3868.

(42) Srebro, M.; Autschbach, J. Does a Molecule-Specific Density Functional Give an Accurate Electron Density? The Challenging Case of the $\mathrm{CuCl}$ Electric Field Gradient. J. Phys. Chem. Lett. 2012, 3, 576581 .

(43) Egger, D. A.; Weissman, S.; Refaely-Abramson, S.; Sharifzadeh, S.; Dauth, M.; Baer, R.; Kümmel, S.; Neaton, J. B.; Zojer, E.; Kronik, L. Outer-valence Electron Spectra of Prototypical Aromatic Heterocycles from an Optimally Tuned Range-Separated Hybrid Functional. J. Chem. Theory Comput. 2014, 10, 1934-1952.

(44) Rohrdanz, M. A.; Martins, K. M.; Herbert, J. M. A long-rangecorrected density functional that performs well for both ground-state properties and time-dependent density functional theory excitation energies, including charge-transfer excited states. J. Chem. Phys. 2009, $130,054112-054119$.

(45) Livshits, E.; Baer, R. A well-tempered density functional theory of electrons in molecules. Phys. Chem. Chem. Phys. 2007, 9, 29322941.

(46) Chai, J.-D.; Head-Gordon, M. Long-range corrected hybrid density functionals with damped atom-atom dispersion corrections. Phys. Chem. Chem. Phys. 2008, 10, 6615-6620.

(47) Perdew, J. P.; Parr, R. G.; Levy, M.; Balduz, J. L. DensityFunctional Theory for Fractional Particle Number: Derivative Discontinuities of the Energy. Phys. Rev. Lett. 1982, 49, 1691-1694.

(48) Almbladh, C.-O.; von Barth, U. Exact results for the charge and spin densities, exchange-correlation potentials, and density-functional eigenvalues. Phys. Rev. B: Condens. Matter Mater. Phys. 1985, 31, $3231-3244$. 
(49) Perdew, J. P.; Levy, M. Comment on 'Significance of the highest occupied Kohn-Sham eigenvalue'. Phys. Rev. B: Condens. Matter Mater. Phys. 1997, 56, 16021-16028.

(50) Levy, M.; Perdew, J. P.; Sahni, V. Exact differential equation for the density and ionization energy of a many-particle system. Phys. Rev. A: At., Mol., Opt. Phys. 1984, 30, 2745-2748.

(51) Stein, T.; Kronik, L.; Baer, R. Prediction of charge-transfer excitations in coumarin-based dyes using a range-separated functional tuned from first principles. J. Chem. Phys. 2009, 131, 244119-244123.

(52) Stein, T.; Autschbach, J.; Govind, N.; Kronik, L.; Baer, R. Curvature and Frontier Orbital Energies in Density Functional Theory. J. Phys. Chem. Lett. 2012, 3, 3740-3744.

(53) Autschbach, J.; Srebro, M. Delocalization Error and "Functional Tuning" in Kohn-Sham Calculations of Molecular Properties. Acc. Chem. Res. 2014, 47, 2592-2602.

(54) Körzdörfer, T.; Brédas, J.-L. Organic Electronic Materials: Recent Advances in the DFT Description of the Ground and Excited States Using Tuned Range-Separated Hybrid Functionals. Acc. Chem. Res. 2014, 47, 3284-3291.

(55) Tamblyn, I.; Refaely-Abramson, S.; Neaton, J. B.; Kronik, L. Simultaneous Determination of Structures, Vibrations, and Frontier Orbital Energies from a Self-Consistent Range-Separated Hybrid Functional. J. Phys. Chem. Lett. 2014, 5, 2734-2741.

(56) Kronik, L.; Kümmel, S. Dielectric screening meets optimallytuned density functionals. Adv. Mater. 2018, 1706560.

(57) Shao, Y.; et al. Advances in molecular quantum chemistry contained in the Q-Chem 4 program package. Mol. Phys. 2015, 113, 184-215.

(58) Dunning, T. H. Gaussian basis sets for use in correlated molecular calculations. I. The atoms boron through neon and hydrogen. J. Chem. Phys. 1989, 90, 1007.

(59) Ihm, J.; Zunger, A.; Cohen, M. L. J. Phys. C: Solid State Phys. 1979, 12, 4409 .

(60) Troullier, N.; Martins, J. L. Phys. Rev. B: Condens. Matter Mater. Phys. 1991, 43, 1993.

(61) ABINIT. http://www.abinit.org/ (accessed May 2018).

(62) Schatschneider, B.; Liang, J.-J.; Reilly, A. M.; Marom, N.; Zhang, G.-X.; Tkatchenko, A. Electrodynamic response and stability of molecular crystals. Phys. Rev. B: Condens. Matter Mater. Phys. 2013, 87, 060104.

(63) Deslippe, J.; Samsonidze, G.; Strubbe, D. A.; Jain, M.; Cohen, M. L.; Louie, S. G. Comput. Phys. Commun. 2012, 183, 1269.

(64) Rohlfing, M.; Louie, S. G. Phys. Rev. B: Condens. Matter Mater. Phys. 2000, 62, 4927.

(65) Sato, N.; Seki, K.; Inokuchi, H. Polarization energies of organic solids determined by ultraviolet photoelectron spectroscopy. J. Chem. Soc., Faraday Trans. 2 1981, 77, 1621-1633.

(66) Neaton, J. B.; Hybertsen, M. S.; Louie, S. G. Renormalization of Molecular Electronic Levels at Metal-Molecule Interfaces. Phys. Rev. Lett. 2006, 97, 216405.

(67) Yang, Z.-h.; Sottile, F.; Ullrich, C. A. Simple screened exactexchange approach for excitonic properties in solids. Phys. Rev. B: Condens. Matter Mater. Phys. 2015, 92, 035202-035206.

(68) Shimazaki, T.; Nakajima, T. Theoretical study of a screened Hartree-Fock exchange potential using position-dependent atomic dielectric constants. J. Chem. Phys. 2015, 142, 074109.

(69) Shimazaki, T.; Nakajima, T. Application of the dielectricdependent screened exchange potential approach to organic photocell materials. Phys. Chem. Chem. Phys. 2016, 18, 27554-27563.

(70) Skone, J. H.; Govoni, M.; Galli, G. Nonempirical rangeseparated hybrid functionals for solids and molecules. Phys. Rev. B: Condens. Matter Mater. Phys. 2016, 93, 235106-235117.

(71) Brawand, N. P.; Vörös, M.; Govoni, M.; Galli, G. Generalization of Dielectric-Dependent Hybrid Functionals to Finite Systems. Phys. Rev. X 2016, 6, 041002-041010.

(72) Phillips, H.; Zheng, Z.; Geva, E.; Dunietz, B. D. Orbital gap predictions for rational design of organic photovoltaic materials. Org. Electron. 2014, 15, 1509-1520.
(73) Sun, H.; Ryno, S.; Zhong, C.; Ravva, M. K.; Sun, Z.; Korzdorfer, T.; Brédas, J.-L. Ionization Energies, Electron Affinities, and Polarization Energies of Organic Molecular Crystals: Quantitative Estimations from a Polarizable Continuum Model (PCM)-Tuned Range-Separated Density Functional Approach. J. Chem. Theory Comput. 2016, 12, 2906-2916.

(74) Zheng, Z.; Egger, D. A.; Brédas, J.-L.; Kronik, L.; Coropceanu, V. Effect of Solid-State Polarization on Charge-Transfer Excitations and Transport Levels at Organic Interfaces from a Screened RangeSeparated Hybrid Functional. J. Phys. Chem. Lett. 2017, 8, 3277.

(75) de Queiroz, T. B.; Kümmel, S. Charge-transfer excitations in low-gap systems under the influence of solvation and conformational disorder: Exploring range-separation tuning. J. Chem. Phys. 2014, 141, 084303.

(76) Warren, S. G. Optical constants of carbon dioxide ice. Appl. Opt. 1986, 25, 2650-2674.

(77) Dressler, K.; Schnepp, O. Absorption Spectra of Solid Methane, Ammonia, and Ice in the Vacuum Ultraviolet. J. Chem. Phys. 1960, 33, 270 .

(78) Donaldson, W. R.; Tang, C. L. Urea optical parametric oscillator. Appl. Phys. Lett. 1984, 44, 25-27.

(79) Landt, L.; Klünder, K.; Dahl, J. E.; Carlson, R. M. K.; Möller, T.; Bostedt, C. Optical Response of Diamond Nanocrystals as a Function of Particle Size, Shape, and Symmetry. Phys. Rev. Lett. 2009, 103, 047402 .

(80) Doering, J. P. Electronic energy levels of benzene below $7 \mathrm{eV}$. J. Chem. Phys. 1999, 67, 4065-4070.

(81) Silinsh, E. A. Organic Molecular Crystals; Springer-Verlag: Berlin, 1980.

(82) Pope, M.; Swenberg, C. E. Electronic Processes in Organic Crystals and Polymers; Oxford University Press: New York, 1999.

(83) Lewis, T. P.; Ragin, H. R. Polarized single-crystal absorption spectra of pyrazine and tetramethylpyrazine. J. Am. Chem. Soc. 1972, 94, 5566-5573.

(84) Aartsma, T. J.; Wiersma, D. A. Stark and zeeman effects on the lower electronic states of s-triazine. Chem. Phys. 1973, 1, 211-216.

(85) Eaton, W. A.; Lewis, T. P. Polarized Single-Crystal Absorption Spectrum of 1-Methyluracil. J. Chem. Phys. 1970, 53, 2164-2172.

(86) Lewis, T. P.; Eaton, W. A. Polarized single-crystal absorption spectrum of cytosine monohydrate. J. Am. Chem. Soc. 1971, 93, 20542056.

(87) da Silva, M. B.; Francisco, T. S.; Maia, F. F.; Caetano, E. W. S.; Fulco, U. L.; Albuquerque, E. L.; Freire, V. N. Improved description of the structural and optoelectronic properties of DNA/RNA nucleobase anhydrous crystals: Experiment and dispersion-corrected density functional theory calculations. Phys. Rev. B: Condens. Matter Mater. Phys. 2017, 96, 085206.

(88) Faber, C.; Attaccalite, C.; Olevano, V.; Runge, E.; Blase, X. Firstprinciples $G W$ calculations for DNA and RNA nucleobases. Phys. Rev. B: Condens. Matter Mater. Phys. 2011, 83, 115123.

(89) Marom, N.; Ren, X.; Moussa, J. E.; Chelikowsky, J. R.; Kronik, L. Electronic structure of copper phthalocyanine from $G_{0} W_{0}$ calculations. Phys. Rev. B: Condens. Matter Mater. Phys. 2011, 84, 195143.

(90) Marom, N.; Caruso, F.; Ren, X.; Hofmann, O. T.; Körzdörfer, T.; Chelikowsky, J. R.; Rubio, A.; Scheffler, M.; Rinke, P. Benchmark of $G W$ methods for azabenzenes. Phys. Rev. B: Condens. Matter Mater. Phys. 2012, 86, 245127.

(91) Blase, X.; Attaccalite, C.; Olevano, V. First-Principles GW Calculations for Fullerenes, Porphyrins, Phthalocyanine, and Other Molecules of Interest for Organic Photovoltaic Applications. Phys. Rev. B: Condens. Matter Mater. Phys. 2011, 83, 115103.

(92) Rangel, T.; Berland, K.; Sharifzadeh, S.; Brown-Altvater, F.; Lee, K.; Hyldgaard, P.; Kronik, L.; Neaton, J. B. Structural and excited-state properties of oligoacene crystals from first principles. Phys. Rev. B: Condens. Matter Mater. Phys. 2016, 93, 115206.

(93) Sharifzadeh, S.; Darancet, P.; Kronik, L.; Neaton, J. B. LowEnergy Charge-Transfer Excitons in Organic Solids from First- 
Principles: The Case of Pentacene. J. Phys. Chem. Lett. 2013, 4, 2197-

2201.

(94) Sharifzadeh, S.; Wong, C. Y.; Wu, H.; Cotts, B. L.; Kronik, L.;

Ginsberg, N. S.; Neaton, J. B. Relating the Physical Structure and

Optoelectronic Function of Crystalline TIPS-Pentacene. Adv. Funct.

Mater. 2015, 25, 2038-2046. 